\title{
A Preliminary Estimate of the Number of People who Inject Drugs in Cherokee Nation using Capture- Recapture Methods
}

\author{
Molly Feder ( $\square$ mfeder@cardeaservices.org ) \\ Cardea Services https://orcid.org/0000-0002-4254-5151
}

\section{Amanda Winters}

Cardea Services

\section{Whitney Essex}

Cherokee Nation Health Services

\section{Jorge Mera}

Cherokee Nation Health Services

\section{Research article}

Keywords: People Who Inject Drugs, Injection Drug Use, American Indian, Alaska Native, CaptureRecapture

Posted Date: January 15th, 2021

DOl: https://doi.org/10.21203/rs.3.rs-144638/v1

License: (9) This work is licensed under a Creative Commons Attribution 4.0 International License. Read Full License 


\section{Abstract}

Background: Injection drug use is an important public health issue in the United States, and estimates indicate that American Indian and Alaska Native people are disproportionately affected. Injection drug use is also the leading cause of Hepatitis $\mathrm{C}$ virus (HCV) infection in the United States, attributable to over half of all cases, and contributes to $44 \%$ of human immunodeficiency virus (HIV) acquisition in American Indian and Alaska Native females. Existing estimates of American Indian and Alaska Native people who inject drugs are limited. We aimed to estimate the number of people who inject drugs in Cherokee Nation.

Methods: A two-sample, capture-recapture approach was used. The first data source was an abstraction of Cherokee Nation Health Services electronic medical records from February 2017 through December 2018. The second data source was an abstraction from Cherokee Nation's HCV Elimination Program Database from August 2015 through December 2018. Individuals were included in the abstractions if they were asked if they had injected drugs in the past six months during their health visit. The indirect prevalence estimate of people who inject drugs was calculated in accordance with the UNAIDS/WHO Guidelines on Estimating the Size of Populations Most at Risk to HIV.

Results: In total, 198 individuals across both data sources reported that they had injected drugs within the past six months. This included 123 unique individuals from the first source, 69 individuals from the second source, and six individuals who were included in both sources. Capture-recapture calculations indicated an estimate of 1,613 people who inject drugs (95\% Cl: $404,2,821)$.

Conclusions: This study was the first attempt at estimating the number of people who inject drugs in Cherokee Nation, and one of the few existing studies to estimate the number of American Indian/Alaska Native people who inject drugs in the United States. Gaining knowledge about the prevalence of people who inject drugs in Cherokee Nation will inform strategies to support addiction care and treatment among people who inject drugs living in Indian Country.

\section{Background}

Increasingly, injection drug use has become recognized as an important public health issue in the United States, and estimates indicate that American Indian and Alaska Native (AI/AN) people are disproportionately affected. In 2018, the Substance Abuse and Mental Health Services Administration (SAMHSA) estimated that $1.8 \%$ of individuals in the United States aged 18 and older had used needles for injecting a drug that was not prescribed, including heroin, cocaine, and methamphetamine, in their lifetime, and that use of injection drugs was higher among AI/AN people (1). During this time, SAMHSA reported that approximately $6.5 \%$ of the $\mathrm{Al} / \mathrm{AN}$ population aged 18 and older, compared with $4.7 \%$ of the non-Hispanic white population, reported opioid use within the last year; and approximately $4.7 \%$ of the $\mathrm{Al} / \mathrm{AN}$ population aged 18 and older, compared with $0.7 \%$ of the non-Hispanic white population, reported methamphetamine use in the past year (1). 
Injection drug use (IDU) contributes to increased spread of bloodborne infections, including hepatitis $\mathrm{C}$ (HCV) and human immunodeficiency virus (HIV), which disproportionately affect Al/AN people $(2,3)$. In addition, IDU is a major risk factor for endocarditis (4-6) and the leading cause of HCV infection in the United States, attributable to over half of all cases $(7,8)$. From 2012-2018, acute HCV cases have increased each year, with the largest increase seen among AI/AN people (9). HIV diagnoses among Al/AN people increased 39\% from 2010 to 2017 and contributed to 44\% of HIV acquisition in $\mathrm{Al} / \mathrm{AN}$ females in 2018 (10).

Although addressing IDU among AI/AN people is key to overall HCV and HIV reduction efforts, research regarding estimates of people who inject drugs (PWID) among AI/AN people is limited. One crosssectional study conducted by Eitle et al. in 2015 estimated that $3.1 \%$ of $\mathrm{Al} / \mathrm{AN}$ young adults injected drugs; however, the study population only included individuals aged 18 to 26 years (11). In 2002, Mitchell et al. found that between $0.5-2.1 \%$ of Al people from two groups in the Southwest United States had injected heroin within their lifetime, but did not assess current use or injection drug use beyond heroin (12). In 2016, Simoni et al. found that $6 \%$ of Al women had ever injected drugs, although this study was conducted in an urban setting through a mail-based survey (13). Finally, in 2016, SAMHSA reported that approximately $1 \%$ of participants over 18 years had experienced lifetime use of needles to inject heroin (14).

In 2015, Cherokee Nation Health Services (CNHS) implemented an HCV Elimination Program to respond to the public health burden of HCV. In 2019, CNHS implemented the Ending the HIV Epidemic Program to respond to the HIV epidemic affecting rural communities in the Southern United States. Harm reduction is an essential component of these programs, and harm reduction interventions may be optimized by knowing the number of PWID who require care.

Estimating the number of PWID will guide interventions by creating a visible need for harm reduction programs. Without knowing the number of PWID who require care, it is difficult to develop HCV and HIV outreach and harm reduction efforts. This study adds to the limited body of knowledge surrounding PWID estimates in $\mathrm{Al} / \mathrm{AN}$ communities in the United States to begin understanding the need for harm reduction services in $\mathrm{Al} / \mathrm{AN}$ communities.

\section{Methods}

\section{Aim and Study Design}

The objective of this study was to generate a preliminary estimate of the number of PWID in Cherokee Nation using capture-recapture methods. To address this aim, a two-sample capture-recapture approach, including retrospective review of two sources of electronic medical record (EMR) data, was used. Capturerecapture methods can be used in epidemiological studies to calculate indirect prevalence estimates among marginalized populations, including PWID (15-20). Implementing capture-recapture methods to estimate the number of PWID includes identifying two or more sources of data on PWID, and comparing 
overlapping individuals across the sources to estimate the total number of PWID in the population, using the formula outlined in Fig. 1 (19).

\section{Setting}

Cherokee Nation is the largest tribal nation in the United States, spanning 14 counties in Oklahoma and includes over 355,000 registered Cherokee citizens (21). CNHS, Cherokee Nation's network of health centers, is the largest tribally operated health system in the United States, receiving over 350,000 patient visits annually at the hospital alone (22). CNHS serves Cherokee Nation citizens and citizens of other federally recognized tribes (22). This study was approved by the Cherokee Nation Institutional Review Board on October 8th, 2018.

\section{Study Data Sources And Participants}

The project team implemented capture-recapture, comparing data collected through CNHS EMRs and the HCV Elimination Program Database. Although all patients included in the HCV Elimination Program Database are also part of the CNHS EMR system, the databases themselves are distinct. All patients that CNHS serves are included in CNHS EMRs, whereas the HCV Elimination Program Database was developed to track HCV specific indicators collected through the Infectious Disease Department.

The CNHS EMR abstraction included all individuals who received health care through one of CNHS' eight rural health centers or hospital between February 2017 through December 2018 who were asked, "Have you injected drugs in the past six months?" during their health visit. The CNHS HCV Elimination Program Database abstraction included patients who reported injecting drugs in the last six months between August 2015 through December 2018. If a patient was asked the question more than once during the study time period, their most recent response was used for analysis. The inclusion dates for the data abstracted from each source represents the full period of time in which the question "Have you injected drugs in the past six months" was implemented in clinical procedures.

\section{Indicators}

Patient-level clinical and demographic variables were abstracted from the data sources to obtain PWID estimates, including injection drug use in the past six months (yes/no), sex, and age.

\section{Analysis}

The indirect prevalence estimate of and confidence interval for PWID in Cherokee Nation were calculated using the formulas presented in Fig. 1, in accordance with the methods presented by Hickman et al and the UNAIDS/WHO Guidelines on Estimating the Size of Populations Most at Risk to HIV $(19,23)$. Inputs 
for the calculations included the number of CNHS patients from EMRs who responded affirmatively to the question asking whether they had injected drugs in the past six months, and the number of patients from the CNHS HCV Elimination Program Database who responded affirmatively to the same question, between August 2015 and December 2018. These datasets were linked using a unique patient identifier to assess overlap in unique patients across the datasets.

All data cleaning and descriptive quantitative analyses were performed using SPSS Version 19 statistical analysis software (24).

\section{Results}

In total, 198 individuals across both data sources reported that they had injected drugs within the past six months. This included 123 unique individuals from the CNHS EMR abstraction, 69 individuals from the HCV Elimination Program Database, and six individuals who were included in both sources.

The CNHS EMR abstraction included 906 total CNHS patients who were asked if they injected drugs in the past six months in CNHS EMRs. Of the 906 patients, 129 (14.2\%) responded "yes". Slightly more than half $(54.7 \%)$ of individuals is this dataset were female, and the age range varied widely. Among those who reported injecting drugs in the past six months, a slightly higher proportion of participants were female (58.1\%), and individuals tended to be a little younger (Table 1 ). 
Table 1

Characteristics of patients included in CNHS EMRs

\begin{tabular}{|c|c|c|}
\hline Characteristics $\mathrm{N}=906$ & $\mathrm{n}$ & $\%$ \\
\hline \multicolumn{3}{|l|}{ All patients who were asked if they had injected drugs in the past six months } \\
\hline \multicolumn{3}{|l|}{ Injected drugs, past six months } \\
\hline Yes & 129 & 14.2 \\
\hline No & 777 & 85.8 \\
\hline \multicolumn{3}{|l|}{ Sex } \\
\hline Female & 496 & 54.7 \\
\hline Male & 410 & 45.3 \\
\hline Age & 905 & \\
\hline $20-30$ & 217 & 24.0 \\
\hline $31-40$ & 211 & 23.3 \\
\hline $41-50$ & 189 & 20.9 \\
\hline $51-60$ & 202 & 22.3 \\
\hline Older than 60 years & 86 & 9.5 \\
\hline Subset of those who reported injecting drugs in the past six months & 129 & \\
\hline \multicolumn{3}{|l|}{ Sex } \\
\hline Female & 75 & 58.1 \\
\hline Male & 54 & 41.9 \\
\hline \multicolumn{3}{|l|}{ Age } \\
\hline $20-30$ & 47 & 36.4 \\
\hline $31-40$ & 34 & 26.4 \\
\hline $41-50$ & 25 & 19.4 \\
\hline $51-60$ & 17 & 13.2 \\
\hline Older than 60 years & 6 & 4.7 \\
\hline
\end{tabular}

The HCV Elimination Program Database included 947 total CNHS patients who were asked whether they had injected drugs in the past six months. Of the 947 patients, 75 (7.9\%) responded "yes". Approximately $60 \%$ of individuals in this dataset were male, and more than one-third (35.5\%) were between 51 and 
60 years old. Among those who reported injecting drugs in the past six months, a slightly lower proportion of participants were male (57.3\%), and individuals tended to be younger, with nearly one-third (32.0\%) of participants aged 31 to 40 years (Table 2 ).

Table 2

Characteristics of patients included in the HCV Elimination Program Database

\begin{tabular}{|c|c|c|}
\hline Characteristics N = 947 & $\mathbf{n}$ & $\%$ \\
\hline \multicolumn{3}{|l|}{ All patients who were asked if they had injected drugs in the past six months } \\
\hline \multicolumn{3}{|l|}{ Injected drugs, past six months } \\
\hline Yes & 75 & 7.9 \\
\hline No & 870 & 91.9 \\
\hline \multicolumn{3}{|l|}{ Sex } \\
\hline Female & 380 & 40.1 \\
\hline Male & 567 & 59.9 \\
\hline \multicolumn{3}{|l|}{ Age } \\
\hline $20-30$ & 101 & 10.7 \\
\hline $31-40$ & 174 & 18.4 \\
\hline $41-50$ & 219 & 23.1 \\
\hline $51-60$ & 336 & 35.5 \\
\hline Older than 60 years & 117 & 12.4 \\
\hline Subset of those who reported injecting drugs in the past six months & 75 & \\
\hline \multicolumn{3}{|l|}{ Sex } \\
\hline Female & 32 & 42.7 \\
\hline Male & 43 & 57.3 \\
\hline \multicolumn{3}{|l|}{ Age } \\
\hline $20-30$ & 18 & 24.0 \\
\hline $31-40$ & 24 & 32.0 \\
\hline $41-50$ & 15 & 20.0 \\
\hline $51-60$ & 15 & 20.0 \\
\hline Older than 60 years & 3 & 4.0 \\
\hline
\end{tabular}


A higher proportion of PWID included in CNHS EMRs were 40 years old or younger and female, compared with PWID included in the HCV Elimination Program Database (Figure 2).

In implementing capture-recapture methods that compare patient health record data collected through CNHS EMRs and CNHS HCV Elimination Program data, results indicated an estimate of approximately 1,613 PWID (95\% Cl: 404, 2,821) (Figure 3).

\section{Discussion}

Using a two-source capture-recapture approach, we generated a preliminary estimate of approximately 1,613 PWID served through CNHS, with a 95\% confidence interval ranging from 404 to 2,821 PWID. This study was the first attempt at estimating the number of PWID in Cherokee Nation, and one of the few existing studies to estimate the number of $\mathrm{Al} / \mathrm{AN}$ people who inject drugs in the United States.

The proportion of reported PWID was high. Among the 906 individuals who were asked if they had injected drugs in the past six months, as reported in the CNHS EMRs, $14.2 \%$ of individuals responded affirmatively. Among the 947 patients who were asked the same question through the HCV Elimination Program, 7.9\% responded affirmatively. Each of these proportions were much higher than existing PWID estimates among Al/AN people in the literature. However, past studies have also focused on particular groups within Al/AN communities, and not the population of PWID as a whole (11-14).

There are multiple limitations to this study design, related to patient population, health systems, data sources, and methods. Research has demonstrated that social desirability biases lead individuals to underreport drug use, and, in particular, injection drug use (25). It is possible that underreporting of injection drug use led to a falsely low estimated number and proportion of PWID within CNHS. Alternatively, although injection drug use in the past six months is intended to be asked across all CNHS patients, most CNHS patients were not asked this question during their visits. Due to the small percentage of total CNHS patients who were asked the question, it is possible that clinicians were selectively asking this question. This may have introduced selection bias, leading to a falsely high estimated number and proportion of PWID within CNHS and violating the capture-recapture assumptions that all individuals included in the databases were correctly classified, truly had an equal, non-zero probability of being captured, and were representative of the true PWID population within CNHS.

Although the two sources used for the capture-recapture estimate were from different databases, the sources are inherently connected because both sources are abstractions from CNHS patient databases and, thus, are only representative of individuals who receive health services through CNHS. In addition, those who test positive for HCV from within a CNHS clinic are later included in the HCV Elimination Program Database, and PWID are more likely than the general population to become infected with HCV. Although PWID-related data are collected separately in these databases, the inherent connection violates the capture-recapture assumption that samples are independent from one another, which may have led to a falsely low PWID estimate. Although this is a key study limitation, it is also a frequently identified 
limitation in capture-recapture studies, as reliance on related institutions or services to provide data sources is common (23).

In addition, this study violates the capture-recapture assumption of homogeneity of the data sources. Individuals are only captured in the HCV Elimination Program Database if they are enrolled in HCV care, whereas all individuals served through CNHS are captured through CNHS EMRs. However, these were the two available data sources that captured injection drug use in Cherokee Nation and violation of homogeneity is common when using healthcare data (26).

Finally, CNHS is not a closed population and the number of PWID served through CNHS is not stagnant. Individuals can register for health services, leave the healthcare system, start injecting drugs, and stop injecting drugs over time. We attempted to reduce the potential bias of this violation of capture-recapture methods by limiting the data abstraction time period for both data sources to one year. However, when we attempted this analysis, the sample size of PWID was too small to conduct the necessary calculations. Even when including all available data on PWID from CNHS EMRs and the HCV Elimination Program Database, the sample sizes of PWID from the two sources were very small.

Although there were limitations, this study adds to the increasing body of research surrounding PWID estimates among indigenous communities in the United States and provides the first attempt at generating a PWID estimate in Cherokee Nation. There is a need for continued assessment of PWID in Cherokee Nation, including utilizing data sources outside of the healthcare system and reducing the length of time for data abstraction as the sample size of PWID recorded across CNHS databases increases, to inform accuracy of estimates. There is also a need to assess the impact of social determinants of equity on injection drug use and infectious disease among $\mathrm{Al} / \mathrm{AN}$ people.

\section{Conclusions}

Gaining knowledge about the prevalence of PWID in Cherokee Nation and other indigenous communities across the United States should inform policy and programmatic investments in culturally responsive, strengths-based substance use treatment and harm reduction efforts among PWID in Cherokee Nation and other Al/AN communities. Understanding the prevalence of PWID in indigenous communities is also foundational to address social determinants of equity among Al/AN people who are disproportionately impacted by bloodborne infections, including HCV and HIV.

\section{Abbreviations}

\section{$\mathrm{Al} / \mathrm{AN}$}

American Indian and Alaska Native

Al

American Indian

CNHS 
Cherokee Nation Health Services

$\mathrm{HCV}$

Hepatitis $\mathrm{C}$ virus

IDU

Injection drug use

PWID

People who inject drugs

RNA

Ribonucleic acid

SAMHSA

Substance Abuse and Mental Health Services Administration

UNAIDS

The Joint United Nations Programme on HIV/AIDS

WHO

World Health Organization

\section{Declarations}

\section{Ethics approval and consent to participate}

\section{This study was approved by the Cherokee Nation Institutional Review Board on October 8 ${ }^{\text {th }}, 2018$.}

\section{Consent for publication}

Not applicable

\section{Availability of data and materials}

The datasets analyzed during the current study are not publicly available to ensure Cherokee Nation has full oversight of any projects using data from Cherokee Nation Health Services but may be made available with permission from Cherokee Nation IRB on reasonable request.

\section{Competing interests}

WE and JM were investigators for a grant that was given to Cherokee Nation Health Services for Hepatitis C Elimination by the Gilead Foundation. The Gilead Foundation did not participate in the design of the study, data collection activities, analysis, interpretation of data, or in writing this manuscript. 


\section{Funding}

Funding for this research project was provided by the Gilead Foundation.

\section{Authors' contributions}

MF made substantial contributions to the design of the work and analyzed and interpreted data, including conducting calculations for PWID estimates. AW was a major contributor in the conception and design of the work, and writing the manuscript. WE was a major contributor in the conception and design of the work, acquisition of data, and writing the manuscript. JM was a major contributor in the conception, design, and oversight of the work, and writing the manuscript. All authors read and approved the final manuscript.

\section{Acknowledgements}

The authors would like to acknowledge Cherokee Nation Health Services leadership, staff, and clients, along with the broader Cherokee community for their support and contributions to this work. In particular, the authors would like to thank Dr. David Gahn from Cherokee Nation Public Health and Wendy Nakatsukasa-Ono from Cardea Services for their support for this project.

\section{References}

1. Bose J, Hedden SL, Lipari RN, Park-Lee E, Tice P. Key substance use and mental health indicators in the United States: Results from the 2017 National Survey on Drug Use and Health (HHS Publication No. SMA 18-5068, NSDUH Series H-53). Rockville, MD: Center for Behavioral Health Statistics and Quality. Substance Abuse and Mental Health Services Administration. 2018.

2. Persons Who Inject Drugs (PWID)s - Infectious Diseases, Opioids and Injection Drug Use. Centers for Disease Control and Prevention. 2018. https://www.cdc.gov/pwid/opioid-use.html\#: :text=A deadly consequence of the,cause heart infections (endocarditis ). Accessed 11 Aug 2020.

3. Harm Reduction. Indian Health Service. https://www.ihs.gov/opioids/harmreduction/. Accessed 11 Aug 2020.

4. Wurcel AG, Anderson JE, Chui KKH, Skinner S, Knox TA, Snydman DR, et al. Increasing infectious endocarditis admissions among young people who inject drugs. Open Forum Infect Dis. 2016;3(3).

5. Cooper HLF, Brady JE, Ciccarone D, Tempalski B, Gostnell K, Friedman SR. Nationwide increase in the number of hospitalizations for illicit injection drug use-related infective endocarditis. Clin Infect Dis. 2007;45(9):1200-3.

6. Murdoch DR, Corey GR, Hoen B, Miró JM, Fowler VG, Bayer AS, Karchmer AW, Olaison L, Pappas PA, Moreillon P, Chambers ST. Clinical presentation, etiology, and outcome of infective endocarditis in the 
21st century: the International Collaboration on Endocarditis-Prospective Cohort Study. Archives of internal medicine. 2009 Mar 9;169(5):463-73.

7. Howard A, Stone K. The global state of harm reduction 2016. London: Harm Reduction International. 2016.

8. Williams IT, Bell BP, Kuhnert W, Alter MJ. Incidence and transmission patterns of acute hepatitis C in the United States, 1982-2006. Archives of internal medicine. 2011 Feb 14;171(3):242-8.

9. Centers for Disease Control and Prevention. Viral Hepatitis Surveillance - United States, 2018. https://www.cdc.gov/hepatitis/statistics/SurveillanceRpts.htm. Published July 2020. Accessed 21 Sep 2020.

10. HIV and American Indians and Alaska Natives. Centers for Disease Control and Prevention. 2020. https://www.cdc.gov/hiv/group/racialethnic/aian/index.html. Accessed 11 Aug 2020.

11. Eitle D, Greene K, Eitle TM. American Indians, substance use, and sexual behavior: do predictors of sexually transmitted infections explain the race gap among young adults? Sex Transm Dis. 2015 Feb;42(2):64-7.

12. Mitchell CM, Beals J, Novins DK, Spicer P. Drug use among two American Indian populations: prevalence of lifetime use and DSM-IV substance use disorders. Drug Alcohol Depend. 2003;69(1):29-41.

13. Simoni JM, Sehgal S, Walters KL. Triangle of risk: urban American Indian women's sexual trauma, injection drug use, and HIV sexual risk behaviors. AIDS Behav. 2004 Mar;8(1):33-45.

14. Center for Behavioral Health Statistics S. Results from the 2016 national survey on drug use and health: Detailed tables.

15. Raag M, Vorobjov S, Uusküla A. Prevalence of injecting drug use in Estonia 2010-2015: A capturerecapture study. Vol. 16, Harm Reduction Journal. BioMed Central Ltd.; 2019.

16. Uusküla A, Rajaleid K, Talu A, Abel-Ollo K, Des Jarlais DC. A decline in the prevalence of injecting drug users in Estonia, 2005-2009. Int J Drug Policy. 2013 Jul;24(4):312-8.

17. Leclerc P, Vandal AC, Fall A, Bruneau J, Roy E, Brissette $S$, et al. Estimating the size of the population of persons who inject drugs in the island of Montreal, Canada, using a six-source capture-recapture model. Drug Alcohol Depend. 2014 Sep;142:174-80.

18. Jones HE, Welton NJ, Ades AE, Pierce M, Davies W, Coleman B, et al. Problem drug use prevalence estimation revisited: Heterogeneity in capture-recapture and the role of external evidence. Addiction. 2016 Mar 1;111(3):438-47.

19. Hickman M, Taylor C, Chatterjee A, et. al. Estimating the prevalence of problematic drug use: a review of methods and their application. Bull Narc. 2002;LIV:15-32.

20. Mastro TD, Kitayaporn D, Weniger BG, Vanichseni S. Estimating the number of HIV-infected injection drug users in Bangkok: A capture-recapture method. Vol. 84, American Journal of Public Health. 1994. 
21. Cherokee Nation. About the Nation. 2018. http://www.cherokee.org/About-The-Nation. Accessed 11 Aug 2020.

22. Cherokee Nation. About Health Services. 2018. http://www.cherokee.org/Services/Health/AboutHealth-Services. Accessed 11 Aug 2020.

23. UNAIDS/WHO Working Group on HIV/AIDS/STI Surveillance. Guidelines on estimating the size of populations at risk for HIV. Geneva: UNAIDS. 2010.

24. IBM SPSS Statistics for Windows. Armonk, NY: IBM Corp.; 2010.

25. Johnson TP. Sources of Error in Substance Use Prevalence Surveys. Int Sch Res Not. 2014;2014:121.

26. Hook EB, Regal RR. Capture-recapture methods in epidemiology: methods and limitations. Epidemiologic reviews. 1995 Jan 1;17(2):243-64.

\section{Figures}

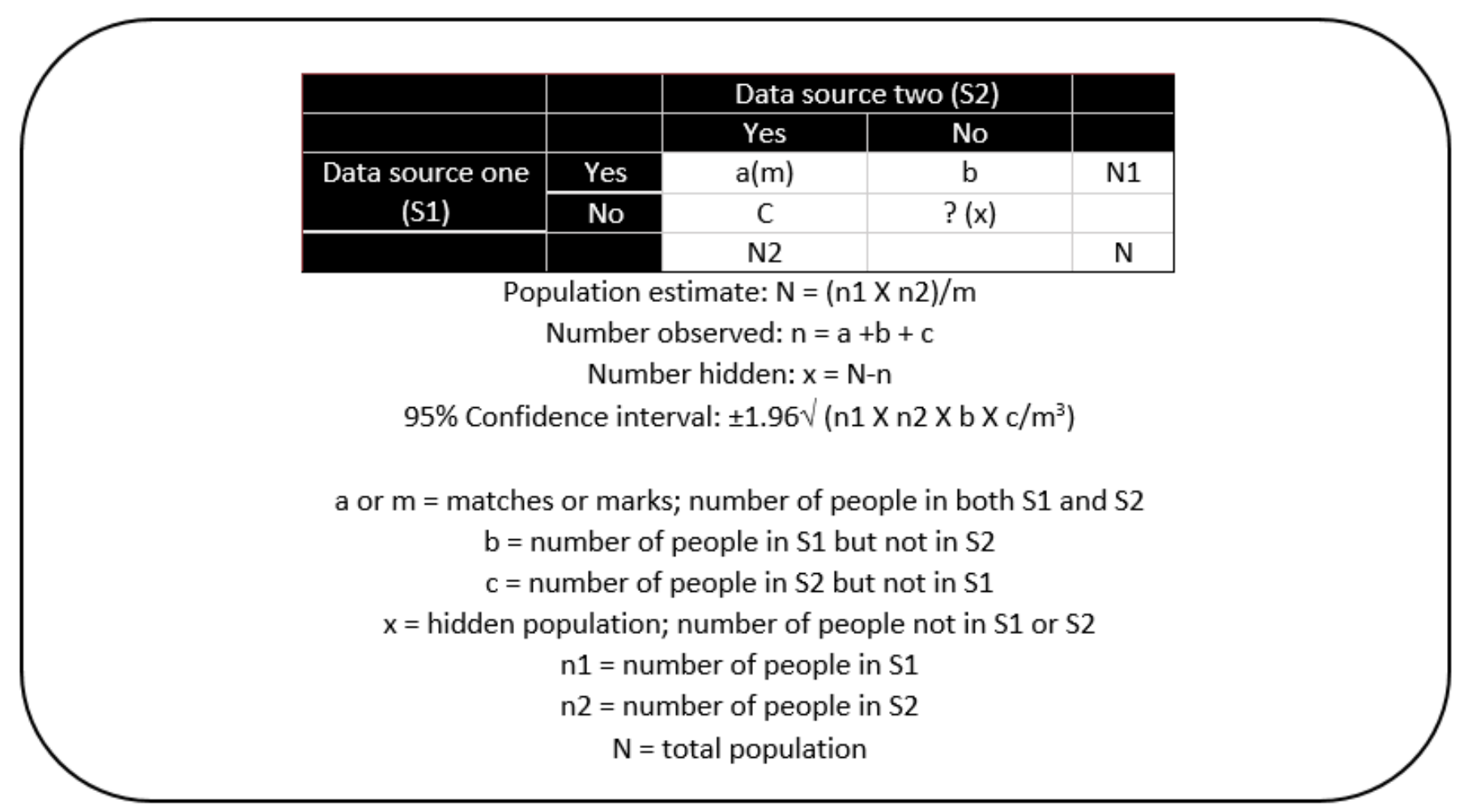

\section{Figure 1}

Formula for attaining PWID prevalence estimates using capture-recapture method with two data sources 

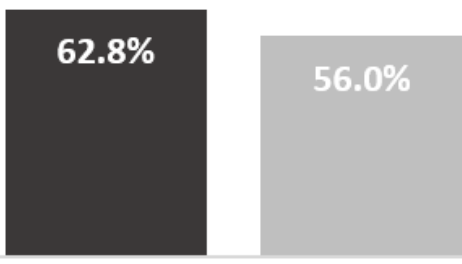

40 years or younger

- PWID in CNHS EMRs ( $n=129)$

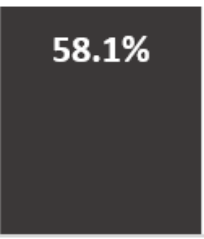

Female
$42.7 \%$

$42.7 \%$

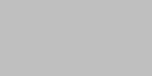

\section{Figure 2}

More PWID in EMRs were young and female compared with the HCV Elimination Program Database

\begin{tabular}{|c|c|c|c|c|}
\hline & & \multicolumn{2}{|c|}{ CNHS HCV Elimination Program Data } & \\
\hline & & Yes & No & \\
\hline \multirow[t]{3}{*}{ CNHS EMR Data } & Yes & 6 & 123 & 129 \\
\hline & No & 69 & ? & \\
\hline & & 75 & & $\mathrm{~N}$ \\
\hline
\end{tabular}

Population estimate: $(129 \times 75) / 6=1,612.5$

Number observed: $6+123+69=198$

Number hidden: $1612.5-198=1,414.5$

$95 \%$ Confidence interval: $\pm 1.96 \sqrt{ }\left(129 \times 75 \times 123 \times 69 / 6^{\wedge} 3\right)= \pm 1,208.46$

PWID estimate $=1,612.5$ (95\% Cl: 404.04, 2,820.96)

\section{Figure 3}

Estimating PWID in CNHS using capture-recapture calculation with two data sources 